\title{
Readmission after rectal resection in the ERAS-era: is a loop ileostomy the Achilles heel?
}

\author{
Johanna Van Butsele, Gabriele Bislenghi, André D'Hoore and Albert M. Wolthuis* (1)
}

\begin{abstract}
Background: Rectal resection surgery is often followed by a loop ileostomy creation. Despite improvements in surgical technique and development of enhanced recovery after surgery (ERAS) protocols, the readmission-rate after rectal resection is still estimated to be around 30\%. The purpose of this study was to identify risk factors for readmission after rectal resection surgery. This study also investigated whether elderly patients ( $\geq 65$ years old) dispose of a distinct patient profile and associated risk factors for readmission.

Methods: This is a retrospective study of prospectively collected data from patients who consecutively underwent rectal resection for cancer within an ERAS protocol between 2011 and 2016. The primary study endpoint was 90-day readmission. Patients with and without readmission within 90 days were compared. Additional subgroup analysis was performed in patients $\geq 65$ years old.
\end{abstract}

Results: A total of 344 patients were included, and $25 \%(n=85)$ were readmitted. Main reasons for readmission were acute renal insufficiency (24\%), small bowel obstruction (20\%), anastomotic leakage (15\%) and high output stoma (11\%). In multivariate logistic regression, elevated initial creatinine level (cut-off values: $0.67-1.17 \mathrm{mg} / \mathrm{dl})$ (OR 1.95, $p=0.041)$ and neoadjuvant radiotherapy (OR 2.63, $p=0.031)$ were significantly associated with readmission. For ileostomy related problems, elevated initial creatinine level (OR 2.76, $p=0.021)$ was identified to be significant.

Conclusion: Recovery after rectal resection within an ERAS protocol is hampered by the presence of a loop ileostomy. ERAS protocols should include stoma education and high output stoma prevention.

Keywords: Rectal resection, Readmission, lleostomy, Risk factors, ERAS

\section{Background}

A defunctioning ileostomy is often created to optimize postoperative outcome after restorative rectal resection and to reduce the risk of anastomotic leakage [1, 2]. Enhanced recovery after surgery (ERAS) protocols were developed and implemented to improve postoperative recovery [3]. ERAS guidelines consist of pre-, periand postoperative evidence-based treatment measures

*Correspondence: albert.wolthuis@uzleuven.be

Department of Abdominal Surgery, University Hospital Gasthuisberg, Leuven, Belgium aiming to reduce the number of complications and shorten the length of hospital stay [3-6]. Those measures consist among other things of early postoperative refeeding and mobilization, thromboembolic prophylaxis, oral carbohydrates preoperatively, opium-free anesthesia and avoidance of usage of nasogastric tubes. Despite all these efforts, 30- to 60-day readmission rates after restorative rectal resection are still estimated to be around 30\% [7, 8]. Overall long-term morbidity rate after rectal resection has been reported to be $20-30 \%$ (mean follow-up time: $36-85$ months) $[9,10]$. Although a combination of efforts has led to improved recovery and shorter length 
of hospital stay, it is hypothesized that patients with a defunctioning ileostomy have a higher risk of acute renal insufficiency and of readmission. The aim of this study was to identify risk factors for readmission in patients after rectal resection and loop ileostomy creation.

\section{Methods}

A retrospective database survey of prospectively collected data from patients who underwent rectal resection surgery within an ERAS-protocol over a 5-year period was conducted. In short, ERAS-protocol was implemented in 2009 and the following aspects were systematically used: preadmission counseling, no premedication, no nasogastric tube, multimodal perioperative analgesia, prevention of sodium and fluid overload, minimally-invasive approach with short incisions, prevention of hypothermia, thrombo-prophylaxis, routine postoperative mobilization, prevention of nausea and vomiting, early removal of catheters [11]. For rectal resections, all patients underwent mechanical bowel preparation as per hospital protocol. There was no systematic use of carbohydrate drinks (immune-nutritional therapy). Inclusion criteria were adult patients who underwent restorative proctectomy between 2011 and 2016. Exclusion criteria were patients who underwent rectal amputation with permanent colostomy, and urgent operations. Primary study endpoint was 90-day readmission. Two attending surgeons (ADH, AW) operated on these patients following the same principles. In general, ileostomies were performed in patients after neoadjuvant therapy, as per center protocol. Ileostomy-related problems were defined as all complications occurring because of the presence of an ileostomy. Complications such as parastomal skin problems, stoma necrosis (complete or partial), leakage caused by a low lying stoma, stenosis, soma bleeding, granuloma formation, prolapse, and parastomal hernia were recorded in the database. Loss of stoma output secondary to other causes was classified as ileostomy-related problem. High output stoma was defined as a stoma output exceeding $2000 \mathrm{ml} / 24 \mathrm{~h}$. Acute renal insufficiency was defined as a decrease in renal function in the postoperative period, measured by an increase in serum creatinine or a decrease in urine output, or both. Anastomotic leakage was defined as a breach in a surgical join between two hollow viscera, with or without active leak of luminal contents. Readmission was defined as unanticipated need for hospitalization after rectal resection (index operation). Creatinine level was measured during hospital stay of the index operation. Initial creatinine level was the first value during hospital admission. Reference values were $0.51-0.95 \mathrm{mg} / \mathrm{dl}$. Abnormal creatinine was defined as creatinine $>0.95 \mathrm{mg} / \mathrm{dl}$. Additional subgroup analysis was performed in patients $\geq 65$ years old. This study was ethically approved by The Research Ethics Committee UZ/KU Leuven (MP007786).

\section{Statistical analysis}

Mann-Whitney $U$ and Fishers exact tests were used to compare continuous/ordinal and categorical variables, respectively, between patients with and without readmission within 90 days. The discriminative ability (C-index) was reported for each of the considered predictors of readmission $(0.5=$ random prediction, $1=$ perfect discrimination). A multivariable logistic regression model was obtained applying a backward selection strategy with $\mathrm{p}=0.157$ as critical $\mathrm{p}$-value to stay in the model. The use of this critical value corresponds to using the Aikake Information Criterion for model selection. With this criterion we require that the increase in model $\chi^{2}$ has to be larger than two times the degrees of freedom. As an alternative, a stepwise selection procedure was used, yielding the same result. The prediction model obtained after applying a model building approach is overoptimistic, in the sense that it overestimates the future performance in new subjects. An optimism-corrected estimate of the performance was obtained using a bootstrap resampling procedure [12]. A similar approach was used to evaluate relations with the presence of an ileostomy problem within 90 days post discharge. Of note: time until readmission was not predicted, but readmission within 90 days. All analyses have been performed using SAS software, version 9.4 of the SAS System for Windows.

\section{Results}

\section{Patient characteristics}

A total of 344 patients who underwent rectal resection within an ERAS-protocol were included, 163 of which were older than 65 years old. Patient characteristics and operative details are shown in Tables 1 and 2. Mean age was $64 \pm 11$ years, whereas mean age in the elderly population was $73 \pm 6$ years. Older patients and the overall population showed a remarkably similar patient profile. Overall, only one third of the patients were female $(32.9 \%)$. The majority of patients could be categorized in American Society of Anesthesiologists (ASA) category II (67.7\%) and were treated with neoadjuvant therapy $(68 \%)$. Sixty-seven percent $(n=231)$ of the patients received a loop ileostomy. Mean postoperative length of stay was $12 \pm 9$ days (median 9 (IQR 7-14) days). Overall readmission rate was 25\% (85 out of 344 patients). Comparable rates of readmission were found in patients $<65$ and $\geq 65$ years old: $25 \%$ (45 out of 181) and 25\% (40 out of 163), respectively. In univariate analysis, there was a significant difference in rate of treatment with neoadjuvant radiotherapy in 
Table 1 Patient characteristics and operative details

\begin{tabular}{|c|c|c|c|c|}
\hline Characteristic & $\begin{array}{l}\text { Overall } \\
n=344\end{array}$ & $\begin{array}{l}\text { No readmission } \\
n=259\end{array}$ & $\begin{array}{l}\text { Readmission } \\
\mathrm{n}=85\end{array}$ & $\mathrm{p}$ value \\
\hline Age (mean $\pm S D)$ & $63.8 \pm 11.4$ & $63.9 \pm 11$ & $63.3 \pm 12.7$ & 0.876 \\
\hline \multicolumn{5}{|l|}{ Gender } \\
\hline Male & $231(67.2 \%)$ & $175(67.6 \%)$ & $56(65.9 \%)$ & \multirow[t]{2}{*}{0.791} \\
\hline Female & 113 (32.9\%) & $84(32.4 \%)$ & $29(34.1 \%)$ & \\
\hline Weight (mean \pm SD) & $77.8 \pm 16.4$ & $77.7 \pm 16.3$ & $78.1 \pm 16.7$ & 0.499 \\
\hline $\mathrm{BMI}($ mean $\pm \mathrm{SD})$ & $26.5 \pm 4.9$ & $26.4 \pm 4.9$ & $26.6 \pm 4.6$ & 0.517 \\
\hline \multicolumn{5}{|l|}{ ASA class } \\
\hline । & 31 (9\%) & $27(10.4 \%)$ & $4(4.7 \%)$ & \multirow[t]{4}{*}{0.179} \\
\hline$\|$ & $233(67.7 \%)$ & $168(64.9 \%)$ & $65(76.4 \%)$ & \\
\hline III & 79 (23\%) & $63(24.3 \%)$ & $16(18.8 \%)$ & \\
\hline IV & $1(0.3 \%)$ & $1(0.4 \%)$ & $0(0 \%)$ & \\
\hline \multicolumn{5}{|l|}{ Smoking behavior } \\
\hline Never & $186(54.2 \%)$ & 141 (54.7\%) & $45(52.9 \%)$ & \multirow[t]{3}{*}{0.818} \\
\hline Stopped smoking & $116(33.8 \%)$ & $85(33 \%)$ & $31(36.5 \%)$ & \\
\hline Actual smoker & 41 (12\%) & $32(12.4 \%)$ & $9(10.6 \%)$ & \\
\hline Charlson comorbidity index (mean $\pm S D$ ) & $4.9 \pm 2.1$ & $4.9 \pm 2.1$ & $4.9 \pm 2$ & 0.785 \\
\hline \multicolumn{5}{|l|}{ Initial creatinine } \\
\hline Abnormal & $66(19.2 \%)$ & $45(17.4 \%)$ & $21(24.7 \%)$ & \multirow[t]{2}{*}{0.154} \\
\hline Normal & $278(80.8 \%)$ & $214(82.6 \%)$ & $64(75.3 \%)$ & \\
\hline \multicolumn{5}{|l|}{ Neoadjuvant therapy } \\
\hline No & $110(32 \%)$ & $87(33.6 \%)$ & $23(27.1 \%)$ & \multirow[t]{4}{*}{0.061} \\
\hline Chemotherapy & $10(2.9 \%)$ & $7(2.7 \%)$ & $3(3.5 \%)$ & \\
\hline Radiotherapy & $32(9.3 \%)$ & $18(7 \%)$ & $14(16.5 \%)$ & \\
\hline Chemoradiotherapy & $192(55.8 \%)$ & $147(56.8 \%)$ & $45(52.9 \%)$ & \\
\hline \multicolumn{5}{|l|}{ Mode of surgery } \\
\hline Open & $66(19.2 \%)$ & $50(19.3 \%)$ & $16(18.8 \%)$ & \multirow[t]{3}{*}{0.615} \\
\hline Open converted & $28(8.1 \%)$ & $19(7.3 \%)$ & $9(10.6 \%)$ & \\
\hline Laparoscopic & $250(72.7 \%)$ & 190 (73.4\%) & $60(70.6 \%)$ & \\
\hline \multicolumn{5}{|l|}{ Additional surgery } \\
\hline No & $318(92.4 \%)$ & $241(93.1 \%)$ & $77(90.6 \%)$ & \multirow[t]{2}{*}{0.480} \\
\hline Yes & $26(7.6 \%)$ & $18(7 \%)$ & $8(9.4 \%)$ & \\
\hline \multicolumn{5}{|l|}{ Ileostoma } \\
\hline No & $113(32.9 \%)$ & $83(32.1 \%)$ & $30(35.3 \%)$ & \multirow[t]{3}{*}{0.697} \\
\hline Already present & $1(0.3 \%)$ & $1(0.4 \%)$ & $0(0 \%)$ & \\
\hline Newly placed & $230(66.9 \%)$ & $175(67.6 \%)$ & $55(64.7 \%)$ & \\
\hline Duration surgery $(\mathrm{h})($ mean $\pm S D)$ & $3.3 \pm 0.9$ & $3.3 \pm 0.8$ & $3.3 \pm 1$ & 0.844 \\
\hline Blood loss (dl) (mean \pm SD) & $2.7 \pm 3.4$ & $2.6 \pm 3.4$ & $2.9 \pm 3.4$ & 0.503 \\
\hline Length of stay (mean $\pm S D$ ) & $12.1 \pm 9.3$ & $12 \pm 9.9$ & $12.3 \pm 7.1$ & 0.104 \\
\hline Creatinine at discharge (mean $\pm S D)$ & $0.9 \pm 0.3$ & $0.9 \pm 0.3$ & $0.9 \pm 0.3$ & 0.808 \\
\hline
\end{tabular}

the patient population older than 65 years old between the readmitted and non-readmitted group (30\% vs. $9.8 \%$ respectively, $\mathrm{p}=0.005)$. No difference was found in readmission rates between patients who did and did not receive a loop ileostomy. There were no patients lost to follow-up.

\section{Prediction of readmission}

Figure 1 shows that $18.3 \%(14.9-22.4 \%, 95 \% \mathrm{CI})$ of patients were readmitted within 30 days after discharge, $21.2 \%(17.7-25.4 \%, 95 \% \mathrm{CI})$ within 60 days after discharge and $24.7 \%(21.0-28.9 \%$, 95\% CI) within 90 days after discharge. Furthermore, mean duration of readmission was $9 \pm 9$ days. 
Table 2 Patient characteristics and operative details in patients $>65$ years old

\begin{tabular}{|c|c|c|c|c|}
\hline Characteristic & $\begin{array}{l}\text { Age }>65 \text { years } \\
n=163\end{array}$ & $\begin{array}{l}\text { No readmission } \\
n=123\end{array}$ & $\begin{array}{l}\text { Readmission } \\
n=40\end{array}$ & p value \\
\hline Age (mean $\pm S D)$ & $73.1 \pm 6.1$ & $72.8 \pm 6.2$ & $74.1 \pm 5.7$ & 0.186 \\
\hline \multicolumn{5}{|l|}{ Gender } \\
\hline Male & 119 (73\%) & $90(73.2 \%)$ & $29(72.5 \%)$ & \multirow[t]{2}{*}{1.000} \\
\hline Female & 44 (27\%) & $33(26.8 \%)$ & $11(27.5 \%)$ & \\
\hline Weight (mean \pm SD) & $78.2 \pm 15$ & $78 \pm 14.8$ & $79 \pm 15.8$ & 0.399 \\
\hline $\mathrm{BMI}($ mean $\pm \mathrm{SD})$ & $27.2 \pm 4.7$ & $27.1 \pm 4.7$ & $27.8 \pm 4.6$ & 0.188 \\
\hline \multicolumn{5}{|l|}{ ASA class } \\
\hline I & $6(3.7 \%)$ & $6(4.9 \%)$ & $0(0 \%)$ & \multirow[t]{3}{*}{0.497} \\
\hline$\|$ & 103 (63.2\%) & $76(61.8 \%)$ & $27(67.5 \%)$ & \\
\hline III & $54(33.1 \%)$ & 41 (33.3\%) & $13(32.5 \%)$ & \\
\hline \multicolumn{5}{|l|}{ IV } \\
\hline \multicolumn{5}{|l|}{ Smoking behavior } \\
\hline Never & 83 (50.9\%) & $64(52 \%)$ & $19(47.5 \%)$ & \multirow[t]{3}{*}{0.772} \\
\hline Stopped smoking & $69(42.3 \%)$ & $50(40.7 \%)$ & $19(47.5 \%)$ & \\
\hline Actual smoker & $11(6.8 \%)$ & $9(7.3 \%)$ & $2(5 \%)$ & \\
\hline Charlson comorbidity index (mean $\pm S D$ ) & $5.9 \pm 1.8$ & $5.8 \pm 1.9$ & $6 \pm 1.5$ & 0.173 \\
\hline \multicolumn{5}{|l|}{ Initial creatinine } \\
\hline Abnormal & $39(23.9 \%)$ & $26(21.1 \%)$ & $13(32.5 \%)$ & \multirow[t]{2}{*}{0.199} \\
\hline Normal & $124(76.1 \%)$ & $97(78.9 \%)$ & $27(67.5 \%)$ & \\
\hline \multicolumn{5}{|l|}{ Neoadjuvant therapy } \\
\hline No & $60(36.8 \%)$ & $49(39.8 \%)$ & $11(27.5 \%)$ & \multirow[t]{4}{*}{0.005} \\
\hline Chemotherapy & $3(1.8 \%)$ & $1(0.8 \%)$ & $2(5 \%)$ & \\
\hline Radiotherapy & $24(14.7 \%)$ & $12(9.8 \%)$ & $12(30 \%)$ & \\
\hline Chemoradiotherapy & $76(46.6 \%)$ & $61(49.6 \%)$ & $15(37.5 \%)$ & \\
\hline \multicolumn{5}{|l|}{ Mode of surgery } \\
\hline Open & $31(19 \%)$ & $25(20.3 \%)$ & $6(15 \%)$ & \multirow[t]{3}{*}{0.676} \\
\hline Open converted & $13(8 \%)$ & $9(7.3 \%)$ & $4(10 \%)$ & \\
\hline Laparoscopic & $119(73 \%)$ & $89(72.4 \%)$ & $30(75 \%)$ & \\
\hline \multicolumn{5}{|l|}{ Additional surgery } \\
\hline No & $153(93.9 \%)$ & $115(93.5 \%)$ & $38(95 \%)$ & \multirow[t]{2}{*}{1.000} \\
\hline Yes & $10(6.1 \%)$ & $8(6.5 \%)$ & $2(5 \%)$ & \\
\hline \multicolumn{5}{|l|}{ Ileostoma } \\
\hline No & $39(23.9 \%)$ & $30(24.4 \%)$ & $9(22.5 \%)$ & \multirow[t]{3}{*}{1.000} \\
\hline Already present & $1(0.6 \%)$ & $1(0.81 \%)$ & $0(0 \%)$ & \\
\hline Newly placed & $123(75.5 \%)$ & $92(74.8 \%)$ & $31(77.5 \%)$ & \\
\hline Duration surgery (h) (mean \pm SD) & $3.3 \pm 0.9$ & $3.3 \pm 0.7$ & $3.5 \pm 1.1$ & 0.820 \\
\hline Blood loss (dl) (mean \pm SD) & $2.6 \pm 3$ & $2.6 \pm 3.2$ & $2.5 \pm 2.6$ & 0.966 \\
\hline Length of stay (mean $\pm S D$ ) & $12.5 \pm 8.3$ & $12 \pm 8.1$ & $14.2 \pm 8.9$ & 0.142 \\
\hline Creatinine at discharge (mean \pm SD) & $1 \pm 0.3$ & $1 \pm 0.3$ & $1 \pm 0.3$ & 0.589 \\
\hline
\end{tabular}

Main reasons for readmission, together encompassing $70 \%$ of the cases were: acute renal insufficiency (24\%), small bowel obstruction (20\%), anastomotic leakage (15\%) and high output stoma (11\%) (Tables 3 and 4). Multivariate logistic regression analysis was used to determine which factors were associated with readmission. Abnormal initial creatinine and neoadjuvant radiotherapy were identified as significantly associated with readmission in the overall population (resp. $\mathrm{OR}=1.95, \mathrm{p}=0.041$ and $\mathrm{OR}=2.63, \mathrm{p}=0.031$ ) (Table 5).

\section{Prediction of ileostomy problems}

Patients who suffered from an ileostomy-related problem were older than patients who did not: mean 
Table 3 Reasons for readmission

\begin{tabular}{|c|c|c|c|c|}
\hline Variable & $\begin{array}{l}\text { Overall } \\
n=344\end{array}$ & $\begin{array}{l}\text { No readmission } \\
n=259\end{array}$ & $\begin{array}{l}\text { Readmission } \\
\mathrm{n}=\mathbf{8 5}\end{array}$ & $p$ value \\
\hline \multicolumn{5}{|c|}{ Any complication } \\
\hline No & $225(65.4 \%)$ & $173(66.8 \%)$ & $52(61.2 \%)$ & \multirow[t]{2}{*}{0.360} \\
\hline Yes & $119(34.6 \%)$ & $86(33.2 \%)$ & $33(38.8 \%)$ & \\
\hline \multicolumn{5}{|c|}{ Number of complications } \\
\hline 0 & $225(65.4 \%)$ & $173(66.8 \%)$ & $52(61.2 \%)$ & \multirow[t]{6}{*}{0.221} \\
\hline 1 & $81(23.6 \%)$ & $54(20.9 \%)$ & $27(31.8 \%)$ & \\
\hline 2 & $26(7.6 \%)$ & $22(8.5 \%)$ & $4(4.7 \%)$ & \\
\hline 3 & $5(1.5 \%)$ & $5(1.9 \%)$ & $0(0 \%)$ & \\
\hline 4 & $6(1.7 \%)$ & $4(1.5 \%)$ & $2(2.4 \%)$ & \\
\hline 5 & $1(0.3 \%)$ & $1(0.4 \%)$ & $0(0 \%)$ & \\
\hline \multicolumn{5}{|c|}{ Anastomotic leakage } \\
\hline No & $320(93 \%)$ & $241(93 \%)$ & 79 (92.9\%) & \multirow[t]{2}{*}{1.000} \\
\hline Yes & $24(7 \%)$ & $18(7 \%)$ & $6(7.1 \%)$ & \\
\hline \multicolumn{5}{|c|}{ Postoperative bleeding } \\
\hline No & $340(98.8 \%)$ & $255(98.5 \%)$ & $85(100 \%)$ & \multirow[t]{2}{*}{0.576} \\
\hline Yes & $4(1.2 \%)$ & $4(1.5 \%)$ & $0(0 \%)$ & \\
\hline \multicolumn{5}{|c|}{ Postoperative ileus } \\
\hline No & $302(87.8 \%)$ & $226(87.3 \%)$ & 76 (89.4\%) & \multirow[t]{2}{*}{0.704} \\
\hline Yes & $42(12.2 \%)$ & $33(12.7 \%)$ & $9(10.6 \%)$ & \\
\hline \multicolumn{5}{|c|}{ SSI type 1 wound infection } \\
\hline No & $338(98.3 \%)$ & $256(98.8 \%)$ & $82(96.5 \%)$ & \multirow[t]{2}{*}{0.163} \\
\hline Yes & $6(1.7 \%)$ & $3(1.2 \%)$ & $3(3.5 \%)$ & \\
\hline \multicolumn{5}{|c|}{ Urinary retention } \\
\hline No & $321(93.3 \%)$ & $240(92.7 \%)$ & 81 (95.3\%) & \multirow[t]{2}{*}{0.466} \\
\hline Yes & $23(6.7 \%)$ & $19(7.3 \%)$ & $4(4.7 \%)$ & \\
\hline \multicolumn{5}{|c|}{ UTI, urological infection } \\
\hline No & $330(95.9 \%)$ & $249(96.1 \%)$ & 81 (95.3\%) & \multirow[t]{2}{*}{0.754} \\
\hline Yes & $14(4.1 \%)$ & $10(3.9 \%)$ & $4(4.7 \%)$ & \\
\hline \multicolumn{5}{|c|}{ Cardiac complication } \\
\hline No & $338(98.3 \%)$ & $256(98.8 \%)$ & $82(96.5 \%)$ & \multirow[t]{2}{*}{0.163} \\
\hline Yes & $6(1.7 \%)$ & $3(1.2 \%)$ & $3(3.5 \%)$ & \\
\hline \multicolumn{5}{|c|}{ Lung complication } \\
\hline No & $334(97.1 \%)$ & $251(96.9 \%)$ & $83(97.7 \%)$ & \multirow[t]{2}{*}{1.000} \\
\hline Yes & $10(2.9 \%)$ & $8(3.1 \%)$ & $2(2.4 \%)$ & \\
\hline \multicolumn{5}{|c|}{ Renal complication } \\
\hline No & $333(96.8 \%)$ & $250(96.5 \%)$ & $83(97.7 \%)$ & \multirow[t]{2}{*}{1.000} \\
\hline Yes & $11(3.2 \%)$ & $9(3.5 \%)$ & $2(2.4 \%)$ & \\
\hline Catheter a & quired infectic & & & \\
\hline No & $328(95.4 \%)$ & $246(95 \%)$ & $82(96.5 \%)$ & 0.769 \\
\hline Yes & $16(4.7 \%)$ & $13(5 \%)$ & $3(3.5 \%)$ & \\
\hline High outp & ut stoma & & & \\
\hline No & $325(94.5 \%)$ & $247(95.4 \%)$ & 78 (91.8\%) & 0.271 \\
\hline Yes & $19(5.5 \%)$ & $12(4.6 \%)$ & $7(8.2 \%)$ & \\
\hline Small bow & el obstruction & & & \\
\hline No & $341(99.1 \%)$ & $256(98.8 \%)$ & $85(100 \%)$ & 1.000 \\
\hline Yes & $3(0.9 \%)$ & $3(1.2 \%)$ & $0(0 \%)$ & \\
\hline Ileostomy & oroblem & & & \\
\hline No & 314 (91.3\%) & 238 (91.9\%) & 76 (89.4\%) & 0.508 \\
\hline
\end{tabular}

Table 3 (continued)

\begin{tabular}{lcclc}
\hline Variable & $\begin{array}{l}\text { Overall } \\
\mathbf{n}=\mathbf{3 4 4}\end{array}$ & $\begin{array}{l}\text { No readmission } \\
\mathbf{n}=\mathbf{2 5 9}\end{array}$ & $\begin{array}{l}\text { Readmission } \\
\mathbf{n = 8 5}\end{array}$ & p value \\
\hline Yes & $30(8.7 \%)$ & $21(8.1 \%)$ & $9(10.6 \%)$ \\
\hline
\end{tabular}

age $68 \pm 11$ years versus $63 \pm 11$ years, respectively $(\mathrm{p}=0.025)$. Abnormal initial creatinine value $(\mathrm{OR}=2.76$, $\mathrm{p}=0.021$ ) was determined as risk factor for development of ileostomy problems (Table 6).

\section{Discussion}

This study shows that the readmission rate after rectal resection was $25 \%$, and most readmissions occurred within 30 days after discharge. These findings are in line with the literature (Table 7) [6-8, 13-22]. Abnormal initial creatinine and neoadjuvant therapy were identified as significantly associated with readmission. Moreover, most patients were readmitted because of acute renal insufficiency secondary to ileostomyrelated problems. In a similar study, unplanned hospital readmission following ileostomy was $29 \%$. Also, renal impairment at discharge was the most important risk factor to predict readmission [23]. In another recent study, Fielding et al. found that postoperative renal impairment more frequently occurred in patients with a diverting ileostomy. Moreover, ileostomy formation was independently associated with kidney injury, and continued to have an impact, even after stoma closure [24]. Another study from the NSQIP dataset by Kim et al. showed that patients with postoperative renal impairment were much more likely to be readmitted after ileostomy creation [25]. O'Connell et al. identified surgical site infection (SSI) and stoma formation as significant risk factors for readmission in a study with a comparative sample size [26]. This can be attributed to the fact that firstly, SSI rate was much lower in our population (1.7\% versus almost $10 \%)$ and secondly, the conclusion concerning stoma formation in the study by O'Connell et al. was based on seven cases (4/31 in the readmission group, $3 / 215$ in the no-readmission group) [26]. We also observed an increased readmission risk after stoma formation (7/85 in the readmission group, $12 / 259$ in the no-readmission group), although this was not statistically significant. It has already been shown that patients who received a stoma after colorectal resection are more likely to be readmitted to the hospital [7, 27, 28]. Many factors associated with readmission like age and past medical history are not prone to modification. In those high-risk cases, reduction of readmission should be attempted through adequate patient selection and preoperative optimization. The 
Table 4 Reasons for readmission in patients $>65$ years old

\begin{tabular}{|c|c|c|c|c|}
\hline Variable & $\begin{array}{l}\text { Age }>65 \text { years } \\
n=163\end{array}$ & $\begin{array}{l}\text { No readmission } \\
n=123\end{array}$ & $\begin{array}{l}\text { Readmission } \\
n=40\end{array}$ & $p$ value \\
\hline \multicolumn{5}{|c|}{ Any complication } \\
\hline No & $101(62.0 \%)$ & 79 (64.2\%) & $22(55 \%)$ & 0.350 \\
\hline Yes & $62(38 \%)$ & $44(35.8 \%)$ & $18(45 \%)$ & \\
\hline \multicolumn{5}{|c|}{ Number of complications } \\
\hline 0 & $101(62 \%)$ & $79(64.2 \%)$ & $22(55 \%)$ & 0.329 \\
\hline 1 & $41(25.2 \%)$ & $27(22 \%)$ & $14(35 \%)$ & \\
\hline 2 & $14(8.6 \%)$ & $12(9.8 \%)$ & $2(5 \%)$ & \\
\hline 3 & $2(1.2 \%)$ & $2(1.6 \%)$ & $0(0 \%)$ & \\
\hline 4 & $5(3.1 \%)$ & $3(2.4 \%)$ & $2(5 \%)$ & \\
\hline \multicolumn{5}{|l|}{5} \\
\hline \multicolumn{5}{|c|}{ Anastomotic leakage } \\
\hline No & $157(96.3 \%)$ & $118(95.9 \%)$ & $39(97.5 \%)$ & 1.000 \\
\hline Yes & $6(3.7 \%)$ & $5(4.1 \%)$ & $1(2.5 \%)$ & \\
\hline \multicolumn{5}{|c|}{ Postoperative bleeding } \\
\hline No & $161(98.8 \%)$ & $121(98.4 \%)$ & $40(100 \%)$ & 1.000 \\
\hline Yes & $2(1.2 \%)$ & $2(1.6 \%)$ & $0(0 \%)$ & \\
\hline \multicolumn{5}{|c|}{ Postoperative ileus } \\
\hline No & $141(86.5 \%)$ & $105(85.4 \%)$ & $36(90 \%)$ & 0.598 \\
\hline Yes & $22(13.5 \%)$ & $18(14.6 \%)$ & $4(10 \%)$ & \\
\hline \multicolumn{5}{|c|}{ SSI type 1 wound infection } \\
\hline No & $159(97.6 \%)$ & $121(98.4 \%)$ & $38(95 \%)$ & 0.253 \\
\hline Yes & $4(2.5 \%)$ & $2(1.6 \%)$ & $2(5 \%)$ & \\
\hline \multicolumn{5}{|c|}{ Urinary retention } \\
\hline No & $147(90.2 \%)$ & $110(89.4 \%)$ & $37(92.5 \%)$ & 0.763 \\
\hline Yes & $16(9.8 \%)$ & $13(10.6 \%)$ & $3(7.5 \%)$ & \\
\hline \multicolumn{5}{|c|}{ UTI, urological infection } \\
\hline No & $157(96.3 \%)$ & $119(96.8 \%)$ & $38(95 \%)$ & 0.636 \\
\hline Yes & $6(3.7 \%)$ & $4(3.3 \%)$ & $2(5 \%)$ & \\
\hline \multicolumn{5}{|c|}{ Cardiac complication } \\
\hline No & $158(96.9 \%)$ & $121(98.4 \%)$ & $37(92.5 \%)$ & 0.095 \\
\hline Yes & $5(3.1 \%)$ & $2(1.6 \%)$ & $3(7.5 \%)$ & \\
\hline \multicolumn{5}{|c|}{ Lung complication } \\
\hline No & $156(95.7 \%)$ & $118(95.9 \%)$ & $38(95 \%)$ & 0.681 \\
\hline Yes & $7(4.3 \%)$ & $5(4.1 \%)$ & $2(5 \%)$ & \\
\hline \multicolumn{5}{|c|}{ Renal complication } \\
\hline No & 155 (95.1\%) & $117(95.1 \%)$ & $38(95 \%)$ & 1.000 \\
\hline Yes & $8(4.9 \%)$ & $6(4.9 \%)$ & $2(5 \%)$ & \\
\hline \multicolumn{5}{|c|}{ Catheter acquired infection } \\
\hline No & $155(95.1 \%)$ & $117(95.1 \%)$ & $38(95 \%)$ & 1.000 \\
\hline Yes & $8(4.9 \%)$ & $6(4.9 \%)$ & $2(5 \%)$ & \\
\hline \multicolumn{5}{|c|}{ High output stoma } \\
\hline No & $152(93.3 \%)$ & $117(95.1 \%)$ & 35 (87.5\%) & 0.140 \\
\hline Yes & $11(6.8 \%)$ & $6(4.9 \%)$ & $5(12.5 \%)$ & \\
\hline \multicolumn{5}{|c|}{ Small bowel obstruction } \\
\hline No & $163(100 \%)$ & $123(100 \%)$ & $40(100 \%)$ & \\
\hline \multicolumn{5}{|l|}{ Yes } \\
\hline \multicolumn{5}{|c|}{ Ileostomy problem } \\
\hline No & $144(88.3 \%)$ & $111(90.2 \%)$ & 33 (82.5\%) & 0.254 \\
\hline
\end{tabular}

Table 4 (continued)

\begin{tabular}{lllll}
\hline Variable & $\begin{array}{l}\text { Age }>\text { 65 years } \\
\mathbf{n}=\mathbf{1 6 3}\end{array}$ & $\begin{array}{l}\text { No readmission } \\
\mathbf{n}=\mathbf{1 2 3}\end{array}$ & $\begin{array}{l}\text { Readmission } \\
\mathbf{n}=\mathbf{4 0}\end{array}$ & p value \\
\hline Yes & $19(11.7 \%)$ & $12(9.8 \%)$ & $7(17.5 \%)$ & \\
\hline
\end{tabular}

Table 5 Multivariate prediction of 90-day readmission: stepwise multivariate logistic regression model

\begin{tabular}{lcc}
\hline & Odds ratio $\mathbf{( 9 5 \% ~ C l )}$ & p value \\
\hline ASA & & \\
ASA 2 & $3.8(1.1-13.1)$ & 0.049 \\
ASA 3-4 & $2.3(0.6-8.6)$ & 0.036 \\
& & 0.228 \\
Initial creatinine & $2(1.0-3.7)$ & \\
Abnormal & & 0.041 \\
Neoadjuvant therapy & $1.8(0.4-7.5)$ & 0.134 \\
Chemotherapy & $1.1(0.6-1.9)$ & 0.443 \\
Chemoradiotherapy & $2.6(1.1-6.3)$ & 0.831 \\
Radiotherapy & & 0.031 \\
\hline
\end{tabular}

Table 6 Multivariate prediction of 90-day ileostomy problem: stepwise multivariate logistic regression model

\begin{tabular}{lll}
\hline & Odds ratio $(\mathbf{9 5 \%} \mathbf{C l})$ & p value \\
\hline $\begin{array}{lll}\text { Initial creatinine } \\
\text { Abnormal }\end{array}$ & $2.8(1.7-6.5)$ & 0.021 \\
Mode of surgery & & 0.1475 \\
Laparoscopic & $0.4(0.2-1.0)$ & 0.052 \\
Open converted & $0.6(0.1-2.5)$ & 0.454 \\
Ileostomy problem & & \\
Yes & $2.6(0.9-7.6)$ & 0.088 \\
\hline
\end{tabular}

implementation of ERAS guidelines may play a major role in that matter. However, our study shows that despite the implementation of ERAS measures, the risk of readmission remains high in the patient population treated with a loop ileostomy. Therefore, efforts should be made to further reduce this risk. Shaffer et al. observed a $58 \%$ reduction of readmission rates and a more than $80 \%$ reduction in readmission-related costs after implementation of a specific patient followup program [29]. A similar program set up by Nagle et al. also resulted in a significant decrease of readmissions (15.5\% to 0\%) [30]. Shah et al. and Hardiman et al. obtained similar results using an enhanced recovery protocol and a patient self-care checklist, respectively [14, 17]. Iqbal et al. even found that a lack of a social worker involvement in planning for discharge is 
Table 7 Overview of the literature

\begin{tabular}{|c|c|c|c|c|c|}
\hline & Sample size & $\begin{array}{l}\text { Readmission } \\
\text { rate (\%) }\end{array}$ & Reason readmission & Risk factors & Protective factors \\
\hline Li et al. 2017 [13] & 1267 & 12.9 & $\begin{array}{l}\text { Infections (3.4\%) } \\
\text { Small bowel obstruction/ileus } \\
(3.3 \%) \\
\text { Dehydration (38.3\%) }\end{array}$ & $\begin{array}{l}\text { Cardiovascular factors (OR } \\
2.0 \text { ) } \\
\text { Renal comorbidity (OR 2.9) } \\
\text { Preoperative chemo/radio- } \\
\text { therapy (OR 4.0) } \\
\text { Laparoscopic approach (OR } \\
\text { 1.7) } \\
\text { Longer operative time (OR } \\
\text { 1.2) } \\
\text { Due to dehydration: } \\
\text { Chemo/radiotherapy (OR 4.7) } \\
\text { Laparoscopic approach (OR } \\
\text { 2.6) }\end{array}$ & Cancer diagnosis (OR 0.2) \\
\hline Fish et al. 2017 [7] & 407 & 28 & $\begin{array}{l}\text { Dehydration (42\%) } \\
\text { Intraperitoneal infections } \\
\quad(33 \%) \\
\text { Extraperitoneal infections } \\
(29 \%)\end{array}$ & $\begin{array}{l}\text { Clavien-Dindo complication } \\
\text { grade } 3 \text { to } 4 \text { (OR 6.7) } \\
\text { Charlson comorbidity index } \\
\text { (OR } 1.4 \text { per point) } \\
\text { Loop stoma (OR 2.2) }\end{array}$ & $\begin{array}{l}\text { Longer length of stay (OR 0.5) } \\
\text { Age } 65 \text { years or older (OR 0.4) }\end{array}$ \\
\hline Shah et al. 2017 [14] & 707 & 12 & & Ileostomy & Enhanced recovery protocol \\
\hline Wood et al. 2017 & 2876 & 8.2 & $\begin{array}{l}\text { Ileus and nausea/vomiting } \\
(26.1 \%) \\
\text { Intra-abdominal ascess } \\
(23.9 \%) \\
\text { SSI }(11.5 \%)\end{array}$ & $\begin{array}{l}\text { Rectal surgery (OR 1.89) } \\
\text { Stoma formation (OR 1.34) } \\
\text { Reoperation during first } \\
\text { admission (OR 4.60) }\end{array}$ & \\
\hline Justiniano et al. 2018 [8] & 262 & 30 & Dehydration (37\%) & & \\
\hline Hayden et al. 2012 & 154 & 20.1 & & $\begin{array}{l}\text { Use of anti-diarrheals } \\
\text { Neoadjuvant therapy }\end{array}$ & \\
\hline Messaris et al. 2012 [16] & 603 & 16.9 & Dehydration (43.1\%) & $\begin{array}{l}\text { Laparoscopic approach } \\
\text { Lack of epidural aneshtesia } \\
\text { Preoperative use of sterois } \\
\text { Postoperative use of diuretics }\end{array}$ & \\
\hline Hardiman et al. 2016 [17] & 430 & 26 & & & \\
\hline Charak et al. 2018 [18] & 99 & 36 & $\begin{array}{l}\text { Dehydration (39\%) } \\
\text { Infection (33\%) } \\
\text { Obstruction (3\%) }\end{array}$ & & \\
\hline Grahn et al. 2018 & 100 & $19.6-20.4$ & $\begin{array}{l}\text { Dehydration }(5.9-8.2 \%) \\
\text { Acute renal failure events } \\
(3.9-10.2 \%)\end{array}$ & $\begin{array}{l}\text { Weekend discharges to home } \\
\text { (OR 4.5) }\end{array}$ & \\
\hline lqbal et al. 2018 [20] & 86 & 26 & & $\begin{array}{l}\text { Preoperative steroid use } \\
\text { History of diabetes } \\
\text { History of depression } \\
\text { Lack of hospital social worker } \\
\text { or postoperative ostomy } \\
\text { education } \\
\text { Presence of complications } \\
\text { after the index procedure }\end{array}$ & \\
\hline Paquette et al. 2013 [21] & 201 & 17 & & $\begin{array}{l}\text { Age greater than } 50 \\
\text { IPAA }\end{array}$ & \\
\hline Chen et al. 2018 [22] & 8064 & 20.1 & & $\begin{array}{l}\text { ASA class III } \\
\text { Female sex } \\
\text { IPAA } \\
\text { Age }>65 \\
\text { Shortened length of stay } \\
\text { ASA class I to II with IBD } \\
\text { Hypertension }\end{array}$ & \\
\hline
\end{tabular}

associated with the highest risk of readmission of all factors analyzed in their series (OR 5.15) [20]. These data suggest that patient guidance and monitoring could be of utmost importance in the attempt to reduce readmission rates and associated costs in ileostomy patients. The fact that in the present study, readmission 


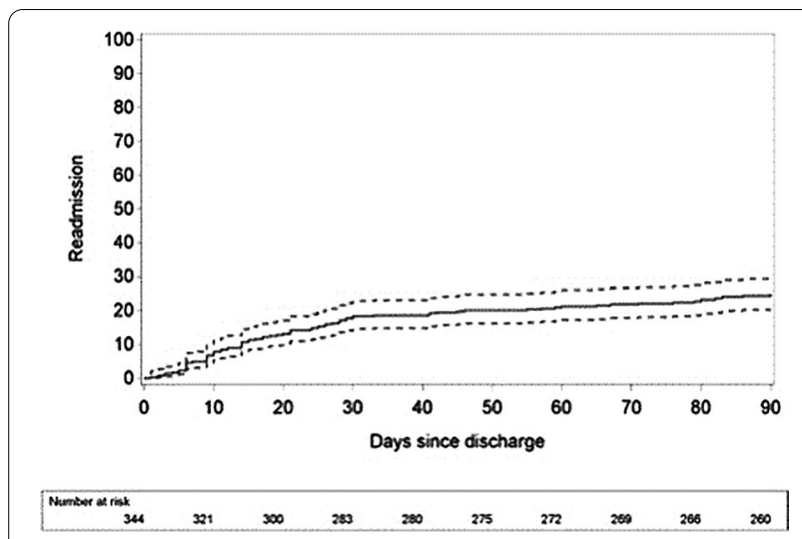

Fig. 1 Readmission rate

\section{Conclusion}

Readmission after rectal resection in the ERAS-era occurs in $25 \%$ of the cases. Most readmissions occur within 30 days after index hospitalization and acute renal insufficiency is frequently associated with readmission. Future patient-education initiatives should be used in conjunction with ERAS guidelines to reduce postoperative readmission.

\section{Abbreviations \\ ASA: American Society of Anesthesiologists; ERAS: Enhanced recovery after surgery; SSI: Surgical site infection; OR: Odds ratio.}

\section{Acknowledgements}

The authors would like to thank Mrs. Isabelle Terrasson and Mrs. Lynn Debrun for their help maintaining the database and following-up the patients.

\section{Authors' contributions}

All authors were involved in study design. JVB and AW analyzed and interpreted the patient data. Feedback on interpretation was given by GB and $\mathrm{ADH}$. JVB was the major contributor in writing the manuscript. All authors agreed to be personally accountable for the work. All authors read and approved the final manuscript.

rate was equal in both age categories is in line with what was reported by Kandagatla et al. [31]. It could be explained that nowadays overall health status, rather than age, influences the postoperative course the most. We also observed that readmission rate did not depend on surgical approach, meaning that presence of an ileostomy was a more important factor. The strengths of our study include a homogenous patient population, consisting of all rectal resection patients and our strict inclusion and exclusion criteria. Our study is unique as it only involves patients who underwent rectal resection and follow-up time is much longer than usual (90day readmission).

The retrospective nature of our study is a potential limitation, as well as the fact that it is a single center study which yielded a limited number of patients. For patients treated within an ERAS protocol, length of hospital stay was rather long. This might be due to the fact that patient's preference regarding discharge plays a role. Unfortunately, data regarding fit for discharge and actual discharge were not available, and could be considered a drawback. Furthermore, patients who were readmitted in outside hospitals were not taken into account and manual analysis of patient files and the use of a coding system was subject to human error. Another limitation of the present study was the lack of information on frailty in older patients and the fact that perioperative fluid balance was not exactly known. Prevention and patient education are key features to avoid readmission secondary to dehydration and ileostomyrelated problems. Currently, a patient-centered protocol and follow-up to detect complications at an early stage via teleconsulting by a specialist nurse are under investigation at our department [32].

\section{Funding \\ There was no funding for this study.}

\section{Availability of data and materials}

The dataset analysed during the current study is available from the corresponding author on reasonable request.

\section{Declarations}

Ethics approval and consent to participate

This study was ethically approved by The Research Ethics Committee UZ/KU Leuven (MP007786).

Not applicable.

\section{Competing interests}

The authors declare that they have no competing interests.

Received: 3 February 2021 Accepted: 10 May 2021

Published online: 27 May 2021

\section{References}

1. Mathiessen P, Hallböök O, Rutegård J, Simert G, Sjödahl R. Defunctioning stoma reduces symptomatic anastomotic leakage after low anterior resection of the rectum for cancer. Ann Surg. 2007;246:207-14.

2. Pisarska M, Gajewska N, Malczak P, Wysocki M, Witowski J, Torbicz G, Major P, Mizera M, Dembinski M, Migaczewski M, Budzynski A, Pedziwiatr M. Defunctioning ileostomy reduces leakage rate in rectal cancer surgery - systematic review and meta-analysis. Oncotarget. 2018:9:20816-25.

3. Forsmo HM, Pfeffer F, Rasdal A, Sintonen H, Körner H, Erichsen C. Preand postoperative stoma education and guidance within an enhanced recovery after surgery (ERAS) programme reduces length of hospital stay in colorectal surgery. Int J Surg. 2016;36:121-6. mission rate in an enhanced recovery after surgery cohort undergoing colorectal cancer surgery. Colorectal Dis. 2018;20:1097-108.

5. Ljungqvist O, Scott M, Fearon KC. Enhanced recovery after surgery: a review. JAMA Surg. 2017;152:292-8.

\section{Consent for publication}

4. Bennedsen ALB, Eriksen JR, Gögenur I. Prolonged hospital stay and read- 
6. Wood T, Aarts MA, Okrainee A, Pearsall E, Victor JC, McKenzie M, Rotstein $\mathrm{O}$, McLeod RS. Emergency room visits and readmissions following implementation of an enhanced recovery after surgery (iERAS) program. J Gastrointest Surg. 2008;22:259-66.

7. Fish DR, Mancuso CA, Garcia-Aguilar JE, Lee SW, Nash GM, Sonoda T, Charlson ME, Temple LK. Readmission after ileostomy creation—retrospective review of a common and significant event. Ann Surg. 2017;265:379-87.

8. Justiniano CF, Temple LK, Swanger AA, Xu Z, Speranza JR, Cellini C, Salloum RM, Fleming FJ. Readmissions with dehydration after ileostomy creation: rethinking risk factors. Dis Colon Rectum. 2018;61:1297-305.

9. Leroy J, Jamali F, Forbes L, Smith M, Rubino F, Mutter D, Marescaux J. Laparoscopic total mesorectal excision (TME) for rectal cancer surgery: long-term outcomes. Surg Endosc. 2004;18:281-9.

10. Contul RB, Grivon M, Fabozzi M, Millo P, Nardi MJ, Aimonetto S, Parini U, Allieta R. Laparoscopic total mesorectal excision for extraperitoneal rectal cancer: long-term results of a 18-year single-center experience. J Gastrointest Surg. 2014;18:796-807.

11. Fierens J, Wolthuis AM, Penninckx F, D'Hoore A. Enhanced recovery after surgery (ERAS) protocol: prospective study of outcome in colorectal surgery. Acta Chir Belg. 2012;112:355-8.

12. Steyerberg E. Clinical prediction models - a practical approach to development, validation, and updating. Berlin: Springer Science + Business Media LLC; 2009.

13. Li W, Stocchi L, Cherla D, Liu G, Agostinelli A, Delaney CP, Steele SR, Gorgun E. Factors associated with hospital readmission following diverting ileostomy creation. Tech Coloproctol. 2017;21:641-8.

14. Shah PM, Johnston L, Sarosiek B, Harrigan A, Friel CM, Thiele RH, Hedrick TL. Reducing readmissions while shortening length of stay: the positive impact of an enhanced recovery protocol in colorectal surgery. Dis Colon Rectum. 2017;60:219-27.

15. Hayden DM, Pinzon MCM, Francescatti AB, Edquist SC, Malczewski MR, Jolley JM, Brand MI, Saclarides TJ. Hospital readmission for fluid and electrolyte abnormalities following ileostomy construction: preventable of unpredictable? J Gastrointest Surg. 2013;17:298-303.

16. Messaris E, Sehgal R, Deiling S, Koltun WA, Stewart D, McKenna K, Poritz LS. dehydration is the most common indicator for readmission after diverting ileostomy creation. Dis Colon Rectum. 2012;55:175-80.

17. Hardiman KM, Reames CD, McLeod MC, Regenbogen SE, Arbor A. Patient autonomy-centered self-care checklist reduces hospital readmissions after ileostomy creation. Surgery. 2016;160:1302-8.

18. Charak G, Kuritzkes BA, Al-Mazrou A, Suradkar K, Valizadeh N, Lee-Kong SA, Feingold DL, Pappou EP. Use of an ACE inhibitor or angiotensin receptor blocker is a major risk factor for dehydration requiring readmission in the setting of a new ileostomy. Int J Colorectal Dis. 2018;33:311-6.

19. Grahn SW, Lowry AC, Osborne MC, Melton GB, Gaertner WB, Vogler SA, Madoff RD, Kwaan MR. System-wide improvement for transitions after ileostomy surgery: can intensive monitoring of protocol compliance decrease readmissions? A randomized trial. Dis Colon Rectum. 2019;62:363-70.

20. Iqbal A, Sakharuk I, Goldstein L, Tan SA, Qiu P, Li Z, Hughes SJ. Readmission after elective ileostomy in colorectal surgery is predictable. JSLS. 2018;22:1-8.

21. Paquette IM, Solan P, Rafferty JF, Ferguson MA, Davis BR. Readmission for dehydration or renal failure after ileostomy creation. Dis Colon Rectum. 2013;56:974-9.

22. Chen SY, Stem M, Cerullo M, Canner JK, Gearhart SL, Bashar S, Fang S, Efron J. Predicting the risk of readmission from dehydration after ileostomy formation: the dehydration readmission after ileostomy prediction score. Dis Colon Rectum. 2018;61:1410-7.

23. Liu C, Bhat S, O'Grady G, Bissett I. Re-admissions after ileostomy formation: a retrospective analysis from a New Zealand tertiary centre. ANZ J Surg. 2020;90:1621-6.

24. Fielding A, Woods R, Moosvi SR, Wharton RQ, Speakman CTM, Kapur S, Shaikh I, Hernon JM, Lines SW, Stearns AT. Renal impairment after ileostomy formation: a frequent event with long-term consequences. Colorectal Dis. 2020;22:269-78.

25. Kim NE, Hall JF. Risk factors for readmission after ileostomy creation: an NSQIP database study. J Gastrointest Surg. 2020;23:1010 (Epub ahead of print).

26. O'Connell EP, Healy V, Fitzpatrick F, Higgins CA, Burke JP, McNamara DA. Predictors of readmission following proctectomy for rectal cancer. Dis Colon Rectum. 2019;62:6.

27. Bliss LA, Maguire LH, Chau Z, Yang CJ, Nagle DA, Chan AT, Tseng JF. Readmission after resections of the colon and rectum: predictors of a costly and common outcome. Dis Colon Rectum. 2015;58:1164-73.

28. Kulaylat AN, Dillon PW, Hollenbeak CS, Stewart DB. Determinants of 30-d readmission after colectomy. J Surg Res. 2015;193:528-35.

29. Shaffer VO, Owi T, Kumarusamy MA, Sullivan PS, Srinivasan JK, Maithel SK, Staley CA, Sweeney JF, Esper G. Decreasing readmission in ileostomy patients. J Am Coll Surg. 2017;224:425-30.

30. Nagle D, Pare T, Keenan E, Marcet K, Tizio S, Poylin V. Ileostomy pathway virtually eliminates readmissions for dehydration in new ostomates. Dis Colon Rectum. 2012;55:12.

31. Kandagatla P, Nikolian VC, Matusko N, Mason S, Regenbogen SE, Hardiman KM. Patient-reported outcomes and readmission after ileostomy creation in older adults. Am Surg. 2018;84:1814-8.

32. Gonella F, Valenti A, Massucco P, Russolillo N, Mineccia M, Fontana AP, Cucco D, Ferrero A. A novel patient-centered protocol to reduce hospital readmissions for dehydration after ileostomy. Updates Surg. 2019;71:515-21.

\section{Publisher's Note}

Springer Nature remains neutral with regard to jurisdictional claims in published maps and institutional affiliations.
Ready to submit your research? Choose BMC and benefit from:

- fast, convenient online submission

- thorough peer review by experienced researchers in your field

- rapid publication on acceptance

- support for research data, including large and complex data types

- gold Open Access which fosters wider collaboration and increased citations

- maximum visibility for your research: over 100M website views per year

At BMC, research is always in progress.

Learn more biomedcentral.com/submissions 